Punjab University Journal of Mathematics (2021),53(8),527-535

https://doi.org/10.52280/pujm.2021.530801

\title{
Several Congruences Related to Harmonic Numbers
}

\author{
Laala Khaldi \\ EDTNLHM Laboratory, Department of Mathematics, ENS, Vieux-Kouba \\ and Department of Mathematics, University of Bouira, 10000 Bouira, Algeria, \\ Email: 1.khaldi@univ-bouira.dz \\ Farid Bencherif \\ USTHB, Faculty of Mathematics, LA3C laboratory, \\ P.O. 32 El Alia 16111 Algiers, Algeria, \\ Email: fbencherif@usthb.dz or fbencherif@yahoo.fr \\ Abdellah Derbal \\ Ecole Normale Supérieure, Department of Mathematics, \\ EDTNLHM Laboratory, BP 92, Vieux Kouba, Algeria, \\ Email: abdebal@yahoo.fr \\ Miloud Mihoubi \\ USTHB, Faculty of Mathematics, RECITS laboratory, \\ P.O. $32 \mathrm{El}$ Alia 16111 Algiers, Algeria, \\ Email: mmihoubi@usthb.dz or miloudmihoubi@gmail.com
}

Received: 26 April, 2021 / Accepted: 17 June, 2021 / Published online: 26 August, 2021

Abstract.: Let $p$ be a prime greater than or equal to 5. In this paper, by using the harmonic numbers and Fermat quotient we establish congruences involving the sums

$$
\sum_{k=1}^{\frac{p-1}{2}}\left(\begin{array}{l}
k \\
r
\end{array}\right) H_{k}, \quad \sum_{k=1}^{\frac{p-1}{2}} \frac{\left(\begin{array}{c}
2 k \\
k
\end{array}\right)^{2}}{16^{k}} H_{k}^{(2)} \text { and } \sum_{k=1}^{\frac{p-1}{2}} \frac{1}{4^{k}}\left(\begin{array}{c}
2 k \\
k
\end{array}\right) H_{k}^{(3)}
$$

For example,

$$
\sum_{k=0}^{\frac{p-1}{2}} \frac{\left(\begin{array}{c}
2 k \\
k
\end{array}\right)^{2}}{16^{k}} H_{k}^{(2)} \equiv 4 E_{2 p-4}-8 E_{p-3}\left(\bmod p^{2}\right),
$$

where $H_{k}^{(m)}$ are the generalized harmonic numbers of order $m$ and $E_{n}$ are Euler numbers.

\section{AMS (MOS) Subject Classification Codes: 11A07; $11 B 68$}

Key Words: Congruences, harmonic numbers, Fermat quotient. 


\section{INTRODUCTION}

Let $\mathbb{N}$ be the set of natural numbers and $\mathbb{N}^{*}$ be the positive natural numbers. The generalized harmonic numbers $H_{n}^{(m)}$ are the rational numbers defined by

$$
H_{0}^{(m)}:=0, \quad H_{n}^{(m)}:=\sum_{k=1}^{n} \frac{1}{k^{m}}, \quad n \in \mathbb{N}^{*}, m \geq 0 .
$$

As usual,

$$
H_{0}:=H_{0}^{(1)}=0, H_{n}:=H_{n}^{(1)}=\sum_{k=1}^{n} \frac{1}{k}, n \in \mathbb{N}^{*} .
$$

In this paper, we define other generalized harmonic numbers (strict) odd multiple

$$
H_{r}(s)=\sum_{1 \leq j_{1}<j_{2}<\ldots<j_{s} \leq r} \frac{1}{\left(2 j_{1}-1\right)\left(2 j_{2}-1\right) \cdots\left(2 j_{s}-1\right)}, r \geq s \geq 1,
$$

It is convenient to set $H_{0}(s)=0$ for $s \geq 0$ and it is seen that $H_{r}(1)=\sum_{i=1}^{r} \frac{1}{2 i-1}, r \geq 1$.

Let $p$ be a prime number and let $\mathbb{Z}_{(p)}$ be the set of rational numbers having denominators co-prime with $p$. Also, for two reduced rational numbers $\frac{N_{1}}{D_{1}}, \frac{N_{2}}{D_{2}} \in \mathbb{Z}_{(p)}$ such that $D_{1}$ and $D_{2}$ are co-prime with $p$, we write $\frac{N_{1}}{D_{1}} \equiv \frac{N_{2}}{D_{2}}(\bmod p)$ to mean that the numerator $N_{1} D_{2}-N_{2} D_{1}$ is divisible by $p$.

In 2017, Meštrović and Andjić [7] obtained that for each prime $p>3$ and $0 \leqslant m \leqslant$ $p-2$ the congruence

$$
\sum_{k=m}^{p-1}\left(\begin{array}{c}
k \\
m
\end{array}\right) H_{k} \equiv \frac{(-1)^{m}}{m+1}\left(1-p H_{m+1}+\frac{p^{2}}{2}\left(H_{m+1}^{2}-H_{m+1}^{(2)}\right)\right)\left(\bmod p^{3}\right),
$$

where $\left(\begin{array}{c}k \\ m\end{array}\right)$ are the binomial coefficients.

In 2003, Rodriguez-Villegas [8] conjectured the congruence

$$
\sum_{k=0}^{\frac{p-1}{2}} \frac{\left(\begin{array}{c}
2 k \\
k
\end{array}\right)^{2}}{(16)^{k}} \equiv(-1)^{\frac{p-1}{2}}\left(\bmod p^{2}\right) \text {. }
$$

This conjecture proved to be true and was improved by Z-W Sun in 2011[11]. The sequence of Bernoulli numbers $\left(B_{n}\right)_{n \geq 0}$ is defined by

$$
B_{0}=1, \quad B_{n}=-\frac{1}{n+1} \sum_{k=0}^{n-1}\left(\begin{array}{c}
n+1 \\
k
\end{array}\right) B_{k}, n \in \mathbb{N}^{*}
$$

The sequence of Euler numbers $\left(E_{n}\right)_{n \geq 0}$ is defined by

$$
E_{0}=1, \quad E_{n}=-\sum_{k=1,2 \mid k}^{n-1}\left(\begin{array}{l}
n \\
k
\end{array}\right) E_{n-k}, n \in \mathbb{N}^{*} .
$$

It is known that $B_{2 n+1}=E_{2 n+1}=0$ for $n \in \mathbb{N}^{*}$. Many other properties can be found in the literature, see for instance [3, Chapter15]. For $a \in \mathbb{Z}_{(p)}$, we denote by $q_{a}=q_{p}(a)$ the 
Fermat quotient defined for a given prime number $p$ by

$$
q_{a}=\frac{a^{p-1}-1}{p} .
$$

In this paper, we exploit some properties of harmonic numbers to establish congruences for sums in terms involving these numbers. Interesting results on this subject can be found in $[5,7]$. Our main results are as follows.

Theorem 1.1. Let $p \neq 3$ be an odd prime number and let $r \in\left\{0,1, \ldots, \frac{p-1}{2}\right\}$. Then

$$
\sum_{k=r}^{\frac{p-1}{2}}\left(\begin{array}{l}
k \\
r
\end{array}\right) H_{k} \equiv \frac{(-1)^{r}\left(\begin{array}{c}
2 r \\
r
\end{array}\right)}{(r+1) 2^{2 r+1}}\left(X_{r, p}-p Y_{r, p}+p^{2} Z_{r, p}\right) \quad\left(\bmod p^{3}\right)
$$

where

$$
\begin{aligned}
& X_{r, p}=\frac{2 r+1}{r+1}-2 q_{2}, \\
& Y_{r, p}=\frac{1}{r+1}+2 q_{2}-q_{2}^{2}+\left(\frac{2 r+1}{r+1}-2 q_{2}\right) H_{r}(1), \\
& Z_{r, p}=q_{2}^{2}-\frac{2}{3} q_{2}^{3}-\frac{7}{12} B_{p-3}+\left(2 q_{2}-q_{2}^{2}+\frac{1}{r+1}\right) H_{r}(1)+\left(\frac{2 r+1}{r+1}-2 q_{2}\right) H_{r}(2) .
\end{aligned}
$$

Theorem 1.2. Let $p>3$ be a prime number. Then

$$
\sum_{k=0}^{\frac{p-1}{2}}(-1)^{k}\left(\begin{array}{c}
\frac{p-1}{2} \\
k
\end{array}\right)\left(\begin{array}{c}
\frac{p-1}{2}+k \\
k
\end{array}\right) H_{k}^{(2)} \equiv 4 E_{2 p-4}-8 E_{p-3}\left(\bmod p^{2}\right) .
$$

Theorem 1.3. Let $p>3$ be a prime number. Then

$$
\sum_{k=0}^{\frac{p-1}{2}}(-1)^{k-1}\left(\begin{array}{c}
\frac{p-1}{2} \\
k
\end{array}\right) H_{k}^{(3)} \equiv-4 q_{2}^{2}(\bmod p) \text {. }
$$

A simple consequence of Theorem 1.3 is given by the following corollary.

Corollary 1.4. For any prime $p>3$ we have

$$
\sum_{k=0}^{\frac{p-1}{2}} \frac{1}{4^{k}}\left(\begin{array}{c}
2 k \\
k
\end{array}\right) H_{k}^{(3)} \equiv 4 q_{2}^{2}(\bmod p)
$$

In the next section, we present some lemmas to be used later. In section three, we show the proofs of the main results.

\section{PReliminaries}

In this section, we first state some basic facts which will be used later.

Lemma 2.1. $[1,6]$ and $[9$, Thm. 5.2(c)] Let $p \neq 3$ be an odd prime number. Then

$$
\begin{aligned}
& H_{\frac{p-1}{2}} \equiv-2 q_{2}(\bmod p), \\
& H_{\frac{p-1}{2}} \equiv-2 q_{2}+p q_{2}^{2}\left(\bmod p^{2}\right)
\end{aligned}
$$


and

$$
H_{\frac{p-1}{2}} \equiv-2 q_{2}+p q_{2}^{2}-\frac{2}{3} p^{2} q_{2}^{3}-\frac{7}{12} p^{2} B_{p-3}\left(\bmod p^{3}\right) .
$$

Lemma 2.2. Let $p \neq 3$ be an odd prime number and let $r \in\left\{0,1, \ldots, \frac{p-1}{2}\right\}$. Then

$$
\begin{aligned}
\left(\begin{array}{c}
\frac{p-1}{2} \\
r
\end{array}\right) & \equiv \frac{(-1)^{r}}{2^{2 r}}\left(\begin{array}{c}
2 r \\
r
\end{array}\right)\left(1-p H_{r}(1)+p^{2} H_{r}(2)\right)\left(\bmod p^{3}\right), \\
\left(\begin{array}{c}
\frac{p-1}{2}+r \\
2 r
\end{array}\right) & \equiv \frac{\left(\begin{array}{c}
2 r \\
r
\end{array}\right)}{(-16)^{r}}\left(\bmod p^{2}\right),
\end{aligned}
$$

and for $r \in\{0,1, \ldots, p-1\}$ we have

$$
\left(\begin{array}{c}
\left\lfloor\frac{p}{6}\right\rfloor+r \\
2 r
\end{array}\right)\left(\begin{array}{c}
2 r \\
r
\end{array}\right) \equiv \frac{\left(\begin{array}{c}
6 r \\
3 r
\end{array}\right)\left(\begin{array}{c}
3 r \\
r
\end{array}\right)}{(-432)^{r}}(\bmod p) .
$$

Proof. For $r=0,(2.5),(2.6)$ and ( 2. 7 ) are true. For $r \in\left\{1,2, \ldots, \frac{p-1}{2}\right\}$, from the definition of the binomial coefficients, we get

$$
\begin{aligned}
\left(\begin{array}{c}
\frac{p-1}{2}+ \\
r
\end{array}\right) & =\frac{1}{r !} \frac{p-1}{2}\left(\frac{p-1}{2}-1\right) \cdots\left(\frac{p-1}{2}-r+1\right) \\
& =\frac{1}{2^{r} r !}(p-1)(p-3)(p-5) \cdots(p-(2 r-1)) \\
& =\frac{1}{2^{r} r !}(-1)(-3) \cdots(-(2 r-1)) \times\left(1-\frac{p}{1}\right)\left(1-\frac{p}{3}\right) \cdots\left(1-\frac{p}{2 r-1}\right) \\
& =\frac{(-1)^{r}}{2^{r} r !} \prod_{k=1}^{r}(2 k-1) \prod_{k=1}^{r}\left(1-\frac{p}{2 k-1}\right) \\
& =\frac{(-1)^{r}}{2^{2 r}}\left(\begin{array}{c}
2 r \\
r
\end{array}\right) \prod_{k=1}^{r}\left(1-\frac{p}{2 k-1}\right) \\
& =\frac{(-1)^{r}}{2^{2 r}}\left(\begin{array}{c}
2 r \\
r
\end{array}\right)\left(1-p \sum_{1 \leq i \leq r} \frac{1}{2 i-1}+p^{2} \sum_{1 \leq i<j \leq r} \frac{1}{(2 i-1)(2 j-1)}+\cdots\right) \\
& \equiv \frac{(-1)^{r}}{2^{2 r}}\left(\begin{array}{c}
2 r \\
r
\end{array}\right)\left(1-p H_{r}(1)+p^{2} H_{r}(2)\right)\left(\bmod p^{3}\right)
\end{aligned}
$$

which is the congruence ( 2. 5 ). We also have

$$
\begin{aligned}
\left(\begin{array}{c}
\frac{p-1}{2}+r \\
2 r
\end{array}\right) & =\frac{1}{(2 r) !}\left(\frac{p-1}{2}+r\right)\left(\frac{p-1}{2}+r-1\right) \cdots\left(\frac{p-1}{2}-r+1\right) \\
& =\frac{1}{2^{2 r}(2 r) !}(p+2 r-1)(p+2 r-3) \cdots(p-(2 r-3))(p-(2 r-1)) \\
& =\frac{1}{2^{2 r}(2 r) !}\left(p^{2}-1^{2}\right)\left(p^{2}-3^{2}\right) \cdots\left(p^{2}-(2 r-1)^{2}\right) \\
& \equiv \frac{\left(\begin{array}{c}
2 r \\
r
\end{array}\right)}{(-16)^{r}}\left(\bmod p^{2}\right)
\end{aligned}
$$


which gives the congruence (2.6).

To prove $(2.7)$ let $s \in\{1,5\}$ given by $p \equiv s(\bmod 6)$. Then for $r \in\{1,2, \ldots, p-1\}$

$$
\begin{aligned}
\left(\begin{array}{c}
\left\lfloor\frac{p}{6}\right\rfloor+r \\
2 r
\end{array}\right)\left(\begin{array}{c}
2 r \\
r
\end{array}\right) & =\frac{\left(\frac{p-s}{6}+r\right)\left(\frac{p-s}{6}+r-1\right) \cdots\left(\frac{p-s}{6}-r+1\right)}{(2 r) !} \frac{(2 r) !}{(r !)^{2}} \\
& =\frac{(p+6 r-s)(p+6 r-6-s) \cdots(p-6 r+6-s)}{6^{2 r} \cdot(r !)^{2}} \\
& \equiv(-1)^{r} \frac{(6 r-s)(6 r-6-s) \cdots(6-s) \cdot s(s+6) \cdots(s+6 r-6)}{6^{2 r} \cdot(r !)^{2}} \\
& =\frac{(-1)^{r} \cdot(6 r) !}{(2 \cdot 4 \cdots 6 r)(3 \cdot 9 \cdot 15 \cdots(6 r-3)) \cdot 6^{2 r} \cdot(r !)^{2}} \\
& =\frac{(-1)^{r} \cdot(6 r) !}{2^{3 r} \cdot(3 r) ! \cdot\left(\frac{3^{r} \cdot(2 r) !}{2^{r} \cdot r !}\right) \cdot 36^{r} \cdot(r !)^{2}} \\
& \equiv \frac{\left(\begin{array}{c}
6 r \\
3 r
\end{array}\right)\left(\begin{array}{c}
3 r \\
r
\end{array}\right)}{(-432)^{r}}(\bmod p) .
\end{aligned}
$$

In Lemma 2.2, more specifically in the proofs of the main results of the last section, we will use two combinatorial identities given in [2]. For reader's convenience, we quote them here.

Lemma 2.3. [2, Id. 1.48 and 1.51] For all integers $a, l, n$ and $r$ such that $a \leq n$, we have

$$
\sum_{k=0}^{n}\left(\begin{array}{c}
k+l \\
r
\end{array}\right)=\left(\begin{array}{c}
n+l+1 \\
r+1
\end{array}\right)-\left(\begin{array}{c}
l \\
r+1
\end{array}\right)
$$

and

$$
\sum_{k=a}^{n}\left(\begin{array}{l}
k \\
r
\end{array}\right)=\left(\begin{array}{l}
n+1 \\
r+1
\end{array}\right)-\left(\begin{array}{c}
a \\
r+1
\end{array}\right)
$$

Lemma 2.4. Let be $p \neq 3$ be an odd prime number. Then

$$
\sum_{k=1}^{\frac{p-1}{2}} \frac{(-1)^{k-1}}{k^{2}} \equiv(-1)^{\frac{p-1}{2}}\left(2 E_{2 p-4}-4 E_{p-3}\right)\left(\bmod p^{2}\right)
$$


Proof. The result is true for $p=5$. We can assume that $p>5$. The left hand side of ( 2 . 10 ) can be written as

$$
\begin{aligned}
\sum_{k=1}^{\frac{p-1}{2}} \frac{(-1)^{k-1}}{k^{2}} & =\sum_{k=1}^{\frac{p-1}{2}} \frac{1}{k^{2}}-\sum_{k=1}^{\frac{p-1}{2}} \frac{1+(-1)^{k}}{k^{2}} \\
& =\sum_{k=1}^{\frac{p-1}{2}} \frac{1}{k^{2}}-\sum_{k=1}^{\frac{p-1}{2}} \frac{1+(-1)^{k}}{k^{2}} \\
& =\sum_{k=1}^{\frac{p-1}{2}} \frac{1}{k^{2}}-\frac{1}{2} \sum_{k=1}^{\left\lfloor\frac{p}{4}\right\rfloor} \frac{1}{k^{2}}
\end{aligned}
$$

and from Corollary 5.2 (a) given in [9] and Corollary 3.8 given in [12], we finally get

$$
\sum_{k=1}^{\frac{p-1}{2}} \frac{(-1)^{k-1}}{k^{2}} \equiv \frac{7}{3} p B_{p-3}-\frac{1}{2}\left((-1)^{\frac{p-1}{2}}\left(8 E_{p-3}-4 E_{2 p-4}\right)+\frac{14}{3} p B_{p-3}\right)\left(\bmod p^{2}\right)
$$

which proves (2.10) for $p>5$.

Lemma 2.5. [4] Let $n \in \mathbb{N}^{*}$. Then

$$
\begin{aligned}
\sum_{k=0}^{n}(-1)^{n-k}\left(\begin{array}{l}
n \\
k
\end{array}\right)\left(\begin{array}{c}
n+k \\
k
\end{array}\right) H_{k}^{(2)} & =2 \sum_{k=1}^{n} \frac{(-1)^{k-1}}{k^{2}}, \\
\sum_{k=0}^{n}(-1)^{k-1}\left(\begin{array}{l}
n \\
k
\end{array}\right) H_{k}^{(3)} & =\frac{1}{2 n}\left(H_{n}^{2}+H_{n}^{(2)}\right) .
\end{aligned}
$$

\section{Proofs of the MAin Results}

Proof of Theorem 1.1. By the identity ( 2.9), we obtain

$$
\begin{aligned}
\sum_{k=1}^{\frac{p-1}{2}}\left(\begin{array}{l}
k \\
r
\end{array}\right) H_{k} & =\sum_{k=1}^{\frac{p-1}{2}}\left(\begin{array}{l}
k \\
r
\end{array}\right) \sum_{j=1}^{k} \frac{1}{j} \\
& =\sum_{j=1}^{\frac{p-1}{2}} \frac{1}{j} \sum_{k=j}^{\frac{p-1}{2}}\left(\begin{array}{l}
k \\
r
\end{array}\right) \\
& =\sum_{j=1}^{\frac{p-1}{2}} \frac{1}{j}\left(\left(\begin{array}{c}
\frac{p+1}{2} \\
r+1
\end{array}\right)-\left(\begin{array}{c}
j \\
r+1
\end{array}\right)\right) \\
& =\left(\begin{array}{c}
\frac{p+1}{2} \\
r+1
\end{array}\right) H_{\frac{p-1}{2}}-\sum_{j=1}^{\frac{p-1}{2}} \frac{1}{j}\left(\begin{array}{c}
j \\
r+1
\end{array}\right),
\end{aligned}
$$


by the identity $\left(\begin{array}{c}n \\ r\end{array}\right)=\frac{n}{r}\left(\begin{array}{c}n-1 \\ r-1\end{array}\right)$, we get

$$
\sum_{k=1}^{\frac{p-1}{2}}\left(\begin{array}{l}
k \\
r
\end{array}\right) H_{k}=\left(\begin{array}{c}
\frac{p-1}{2} \\
r
\end{array}\right) \frac{1+p}{2(r+1)} H_{\frac{p-1}{2}}-\frac{1}{r+1} \sum_{j=1}^{\frac{p-1}{2}}\left(\begin{array}{c}
j-1 \\
r
\end{array}\right)
$$

and by ( 2.8 ), the same expression can also be expressed as

$$
\sum_{k=1}^{\frac{p-1}{2}}\left(\begin{array}{l}
k \\
r
\end{array}\right) H_{k}=\left(\begin{array}{c}
\frac{p+1}{2} \\
r+1
\end{array}\right)\left(H_{\frac{p-1}{2}}+\frac{2}{1+p}-\frac{1}{r+1}\right) .
$$

Apply the congruence ( 2.5 ) of Lemma 2.2 to obtain

$$
\begin{aligned}
\sum_{k=1}^{\frac{p-1}{2}}\left(\begin{array}{c}
k \\
r
\end{array}\right) H_{k} & \equiv \frac{(-1)^{r}}{(r+1) 2^{2 r+1}}\left(\begin{array}{c}
2 r \\
r
\end{array}\right)(1+p)\left(1-p H_{r}(1)+p^{2} H_{r}(2)\right) \\
& \times\left(H_{\frac{p-1}{2}}+\frac{2}{1+p}-\frac{1}{r+1}\right)\left(\bmod p^{3}\right)
\end{aligned}
$$

We note that

$(1+p)\left(1-p H_{r}(1)+p^{2} H_{r}(2)\right) \equiv 1-p\left(H_{r}(1)-1\right)+p^{2}\left(H_{r}(2)-H_{r}(1)\right)\left(\bmod p^{3}\right)$, so, by the congruence $\frac{2}{1+p} \equiv 2-2 p+2 p^{2}\left(\bmod p^{3}\right)$ and the congruence $(2.4)$ of Lemma2.1, it follows

$$
\begin{aligned}
\sum_{k=1}^{\frac{p-1}{2}}\left(\begin{array}{c}
k \\
r
\end{array}\right) H_{k} & \equiv \frac{(-1)^{r}}{(r+1) 2^{2 r+1}}\left(\begin{array}{c}
2 r \\
r
\end{array}\right)\left(1-p\left(H_{r}(1)-1\right)+p^{2}\left(H_{r}(2)-H_{r}(1)\right)\right) \\
& \times\left(-2 q_{2}+p q_{2}^{2}-\frac{2}{3} p^{2} q_{2}^{3}-\frac{7}{12} p^{2} B_{p-3}+2-2 p+2 p^{2}-\frac{1}{r+1}\right)\left(\bmod p^{3}\right) .
\end{aligned}
$$

After distributing and simplifying the right hand side of the congruence ( 3.13 ) we get congruence (1.2) of Theorem 1.1, which completes the proof.

Proof of Theorem 1.2. To obtain ( 1. 3 ), it suffices to take $n=\frac{p-1}{2}$ in the identity ( 2. 11 ) of Lemma 2.5 and use Lemma 2.4 .

Remark 3.1. By the identity $\left(\begin{array}{c}n \\ k\end{array}\right)\left(\begin{array}{c}n+k \\ k\end{array}\right)=\left(\begin{array}{c}2 k \\ k\end{array}\right)\left(\begin{array}{c}n+k \\ 2 k\end{array}\right)$ and the congruence (2. 6 ), Theorem 1.2 is reduced

$$
\sum_{k=0}^{\frac{p-1}{2}} \frac{\left(\begin{array}{c}
2 k \\
k
\end{array}\right)^{2}}{16^{k}} H_{k}^{(2)} \equiv 4 E_{2 p-4}-8 E_{p-3}\left(\bmod p^{2}\right) .
$$

Taking $n=\left\lfloor\frac{p}{6}\right\rfloor$ in the identity (2.11) of Lemma 2.5 and by the identity $\left.\left(\left\lfloor\frac{p}{6}\right\rfloor\right\rfloor\right\rfloor\left(\left\lfloor\frac{p}{6}\right\rfloor+k\right)=$ $\left(\begin{array}{c}2 k \\ k\end{array}\right)\left(\begin{array}{c}\left\lfloor\frac{p}{6}\right\rfloor+k \\ 2 k\end{array}\right)$, by the congruence (2.7) and the congruence (2.8) of Lemma 2.6 given in [10], we deduce

$$
\sum_{k=1}^{\left\lfloor\frac{p}{6}\right\rfloor} \frac{\left(\begin{array}{c}
6 k \\
3 k
\end{array}\right)\left(\begin{array}{c}
3 k \\
k
\end{array}\right)}{432^{k}} H_{k}^{(2)} \equiv-20 E_{p-3}(\bmod p) .
$$


Proof of Theorem 1.3. To obtain ( 1. 3 ), it suffices to take $n=\frac{p-1}{2}$ in the identity ( 2 . 12 ) of Lemma 2.5 and use the first congruence of Lemma 2.1 noting that $H_{\frac{p-1}{2}}^{(2)} \equiv 0$ $(\bmod p)$.

\section{Particular CASES of Theorem 1}

In this section, we determine the congruence of $\sum_{k=1}^{\frac{p-1}{2}} k^{\alpha} H_{k}\left(\bmod p^{3}\right)$, for $\alpha \in\{0,1,2\}$.

(i) Taking $r=0$ in the congruence (1.2) and use the fact that $H_{0}(1)=H_{0}(2)=0$ to obtain the congruence

$$
\sum_{k=1}^{\frac{p-1}{2}} H_{k} \equiv \frac{1}{2}-q_{2}-p\left(-\frac{1}{2} q_{2}^{2}+q_{2}+\frac{1}{2}\right)+p^{2}\left(-\frac{1}{3} q_{2}^{3}+\frac{1}{2} q_{2}^{2}-\frac{7}{24} B_{p-3}\right)\left(\bmod p^{3}\right) .
$$

(ii) Taking $r=1$ in the congruence (1.2) to obtain

$$
\sum_{k=1}^{\frac{p-1}{2}} k H_{k} \equiv-\frac{1}{8}\left(X_{1, p}-p Y_{1, p}+p^{2} Z_{1, p}\right) \quad\left(\bmod p^{3}\right)
$$

where

$$
X_{1, p}=\frac{3}{2}-2 q_{2}, \quad Y_{1, p}=2-q_{2}^{2} \text { and } Z_{1, p}=\frac{1}{2}+2 q_{2}-\frac{2}{3} q_{2}^{3}-\frac{7}{12} B_{p-3} .
$$

So, the congruence ( 4.16 ) is to be

$$
\sum_{k=1}^{\frac{p-1}{2}} k H_{k} \equiv-\frac{3}{16}+\frac{1}{4} q_{2}-p\left(\frac{1}{8} q_{2}^{2}-\frac{1}{4}\right)+p^{2}\left(\frac{1}{12} q_{2}^{3}-\frac{1}{4} q_{2}-\frac{1}{16}+\frac{7}{96} B_{p-3}\right)\left(\bmod p^{3}\right)
$$

(iii) Taking $r=2$ in the congruence (1.2) to find

$$
\sum_{k=1}^{\frac{p-1}{2}}\left(\begin{array}{l}
k \\
2
\end{array}\right) H_{k} \equiv \frac{1}{16}\left(X_{2, p}-p Y_{2, p}+p^{2} Z_{2, p}\right)\left(\bmod p^{3}\right)
$$

where

$$
\begin{aligned}
X_{2, p} & =\frac{5}{3}-2 q_{2} \\
Y_{2, p} & =\frac{1}{3}+2 q_{2}-q_{2}^{2}+\left(\frac{5}{3}-2 q_{2}\right) H_{2}(1)=\frac{23}{9}-\frac{2}{3} q_{2}-q_{2}^{2} \\
Z_{2, p} & =q_{2}^{2}-\frac{2}{3} q_{2}^{3}-\frac{7}{12} B_{p-3}+\left(\frac{1}{3}+2 q_{2}-q_{2}^{2}\right) H_{2}(1)+\left(\frac{5}{3}-2 q_{2}\right) H_{2}(2) \\
& =1+2 q_{2}-\frac{1}{3} q_{2}^{2}-\frac{2}{3} q_{2}^{3}-\frac{7}{12} B_{p-3}
\end{aligned}
$$


By substituting $\left(\begin{array}{l}k \\ 2\end{array}\right)=\frac{1}{2} k^{2}-\frac{1}{2} k$ in the right hand side of the congruence ( 4.18 ), it can be written as

$$
\frac{1}{2} \sum_{k=1}^{\frac{p-1}{2}} k^{2} H_{k}-\frac{1}{2} \sum_{k=1}^{\frac{p-1}{2}} k H_{k} \equiv \frac{1}{16}\left(X_{2, p}-p Y_{2, p}+p^{2} Z_{2, p}\right)\left(\bmod p^{3}\right),
$$

from which we get

$$
\sum_{k=1}^{\frac{p-1}{2}} k^{2} H_{k} \equiv \sum_{k=1}^{\frac{p-1}{2}} k H_{k}+\frac{1}{8}\left(X_{2, p}-p Y_{2, p}+p^{2} Z_{2, p}\right)\left(\bmod p^{3}\right) .
$$

In view of ( 4.17) and (4.19), we have

$$
\sum_{k=1}^{\frac{p-1}{2}} k^{2} H_{k} \equiv \frac{1}{48}-p\left(-\frac{1}{12} q_{2}+\frac{5}{72}\right)+p^{2}\left(-\frac{1}{24} q_{2}^{2}+\frac{1}{16}\right)\left(\bmod p^{3}\right) \text {. }
$$

\section{ACKNOWLEDGMENT}

I would like to thank the referees for their useful comments and suggestions.

\section{REFERENCES}

[1] G. M. Eisenstein, Eine neue Gattung zahlentheoretischer Funktionnen, welch von zwei Elementen ahhangen und durch gewisse linear Funktional-Gleichungen definirt werden, Berichte Knigl. Pruss. Akad. Wiss. Berlin, 15 (1850) 36-42.

[2] H. W. Gould, Combinatorial identities: A standardized set of tables listing 500 binomial coefficient summations, Second Edition, viii + 106 pp., Published by the author, Morgantown, W.Va, 1972.

[3] K. Ireland and M. Rosen, A Classical Introduction to Modern Number Theory, Springer-Verlag, New York, 1982.

[4] H. T. Jin and D. K. Du, Abel's lemma and identities on harmonic numbers, Integers, 15 (2015), \#A22.

[5] S. Koparal and N. Ömür, On congruences related to central binomial coefficients, harmonic and Lucas numbers, Turkish J. Math. 40 (2016) 973-985.

[6] E. Lehmer, On congruences involving Bernoulli numbers and the quotients of Fermat and Wilson, Ann. of Math. 39, No. 2 (1938) 350-360.

[7] R. Meštrović and M. Andjić, Certain congruences for harmonic numbers, Mathematica Montisnigri, 38 (2017).

[8] F. Rodriguez-Villegas, Hypergeometric families of Calabi-Yau manifolds, in: Calabi-Yau Varieties and Mirror Symmetry (Toronto, ON, 2001), pp. 223-231, Fields Inst. Commun., 38, Amer. Math. Soc., Providence, RI, 2003.

[9] Z. W. Sun, Congruences concerning Bernoulli numbers and Bernoulli polynomials, Discrete. Appl. Math. 105 (2000) 193-123.

[10] Z. W. Sun, On congruences related to central binomial coefficients, J. Number Theory 131 (2011) 22192238.

[11] Z. W. Sun, Super congruences and Euler numbers, Sci. China Math. 54 (2011) 2509-2535.

[12] Z. H. Sun, Congruences involving Bernoulli and Euler numbers, J. Number Theory 128 (2008) 280-312. 